\title{
CAMBIOS EN EL COMPROMISO DE UNIVERSITARIOS CON PAREJA Y USO DE REDES SOCIALES DURANTE EL COVID-19
}

\author{
Giovanna Escobar-Mota, María José García Ortega, Tamara Alessandra González Güémez, Juliana Martín de la \\ Torre, María Fernanda Padilla de Villasante, Carla Federica Rumbos Quintana y Georgina García-Rodríguez \\ Universidad Anáhuac \\ México
}

\begin{abstract}
RESUMEN
El aislamiento involuntario por Covid-19 incrementó el intercambio en línea con fines románticos por medio de redes sociales (Sierra, 2020). Este estudio se enfocó en la exploración de cambios en las variables de compromiso, satisfacción, inversión y alternativas antes de la pandemia y en la actualidad, así como su relación con el uso de redes sociales. Se trabajó con 391 participantes mexicanos, 56\% de mujeres de 18 a 48 años, estudiantes de licenciatura o posgrado, con al menos un año de relación de pareja, comparando los dos momentos por medio de una prueba $T$ para muestras relacionadas. Los resultados muestran un aumento en la percepción de alternativas, misma que presenta una correlación positiva ante el uso de redes sociales y una disminución en el compromiso y la satisfacción, siendo la inversión la única variable que permaneció estable. Este estudio muestra la importancia de la desconexión física y la conexión virtual, y su efecto en las relaciones de pareja.
\end{abstract}

Palabras Clave:

pareja, compromiso, Covid-19, redes sociales, alternativas, satisfacción, inversión.

\section{CHANGES IN THE COMMITMENT OF UNIVERSITY STUDENTS WITH COUPLE AND THE USE OF SOCIAL NETWORKS DURING COVID-19}

\begin{abstract}
The involuntary isolation by Covid-19, increased the online exchange for romantic purposes through social networks (Sierra, 2020). The present study explored changes in the variables of commitment, satisfaction, investment and alternatives before the pandemic and today, as well as their relationship with the use of social media. We worked with 391 Mexican participants, $56 \%$ of women between 18 and 48 years old, undergraduate or graduate students, with at least one year of relationship, comparing both moments by means of a $T$ test for related samples. The results show an increase in the perception of alternatives, which presents a positive correlation with the use of social media and a decrease in commitment and satisfaction, with investment being the only variable that remained stable. The present study shows the importance of physical disconnection and virtual connection, and their effect on couple relationships.
\end{abstract}

Keywords:

couple, engagement, Covid-19, social media, alternatives, satisfaction, investment

Bitácora del ARtículo:

| Recibido: 8 de Mayo de 2021 | Aceptado: 28 de Junio de 2021 | Publicado en línea: Julio-Diciembre de 2021 | 


\title{
CAMBIOS EN EL COMPROMISO DE UNIVERSITARIOS CON PAREJA Y USO DE REDES SOCIALES DURANTE EL COVID-19
}

\begin{abstract}
Giovanna Escobar-Mota, María José García Ortega, Tamara Alessandra González Güémez, Juliana Martín de la Torre, María Fernanda Padilla de Villasante, Carla Federica Rumbos Quintana y Georgina García-Rodríguez Universidad Anáhuac

México
\end{abstract}

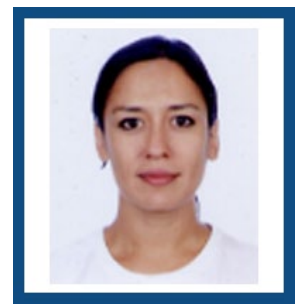

Giovanna Escobar-Mota

Universidad Anáhuac

Correo:gioesmota@gmail.com

Doctora en Psicología Social y Ambiental por la UNM. De 2002 a la fecha ha colaborado en organizaciones no gubernamentales, en distintos niveles y en distintas problemáticas dirigidas a fundaciones nacionales e internacionales. Actualmente realiza investigación básica y aplicada y es docente en instituciones públicas y privadas.

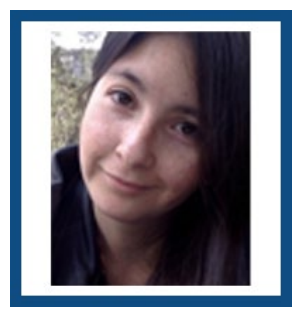

María José García Ortega

Universidad Anáhuac

Correo:maria.garciao@anahuac.mx

Es estudiante de Psicología en la Universidad Anáhuac México, Campus Norte. Participó en el programa de intercambio Youth Rotary International en Sao Paulo, Brasil, como embajadora de la paz y buena voluntad. Ha desarrollado labores como asistente de trabajo de campo para la agencia Ingenum Marketing Communication.

\section{CONTRIBUCIÓN DE LOS AUTORES}

Todos los autores contributeron por igual en la investigación, análisis y redacción.

\section{AGRADECIMIENTOS}

Agradecemos a la Universidad Anahuac México Campus Norte por el acceso al software para realizar los análisis y a nuestra asesora Giovanna Escobar Mota .

\section{DAtos de Filiación de los Autores}

Facultad de Psicología, Universidad Anáhuac México, Campus Norte

\section{(c)

Copyright: (C) 2021 Escobar-Mota, G.; García-Ortega, M.J., González-Güémez, T.A., Martín de la Torre, J., Padilla de Villasante, M.F., Rumbos-Quintana, C.F., y García-Rodríguez, G

Este es un artículo de acceso abierto distribuido bajo los términos de la licencia Creative Commons Reconocimiento-NoComercial 4.0 Internacional, por lo que su contenido gráfico y escrito se puede compartir, copiar y redistribuir total o parcialmente sin necesidad de permiso expreso de sus autoras con la única condición de que no se puede usar con fines directamente comerciales y los términos legales de cualquier trabajo derivado deben ser los mismos que se expresan en la presente declaración. La única condición es que se cite la fuente con referencia a la Revista Digital Internacional de Psicología y Ciencia Social y a sus autoras. 


\section{TABLA DE CONTENIDO}

INTRODUCCIÓN

CONTEXTO ACTUAL: LA PANDEMIA POR COVID-19

POSIBLES EFECTOS EN EL COMPROMISO

MÉTODO

Participantes, 227

Materiales, 227

Procedimiento, 228

Mediciones, 228

Análisis estadísticos, 228

RESULTADOS

Discusión

CONCLUSIONES 


\section{INTRODUCCIÓN}

$\mathbf{L}$ a investigación en relaciones interpersonales ha recopilado evidencia de que son cruciales para nuestro bienestar y se encuentran entre los aspectos que consideramos más importantes en la vida (Perlman y Duck, 1985). Incluso en un mundo donde la naturaleza de las relaciones puede estar cambiando, la pertenencia es un motivo humano universal y clave (Doyle y Molix, 2013). Si bien el estudio de la pareja desde distintas perspectivas -como la antropológica, social y psicológica - no es algo nuevo, cada vez hay más estudios que arrojan luz al entendimiento del papel del amor y sus principales componentes, como el compromiso (Torres-González y Ojeda-García, 2009). Se piensa que el elemento del amor en que las personas más creen es la atracción física; sin embargo, en la estabilidad matrimonial el que tiene mayor peso sigue siendo el compromiso (Rocha-Narváez, Avendaño-Parra, Barrios-Alonso y Polo-Madera, 2017).

Hoy las representaciones y contratos sociosexuales de los jóvenes, sobre todo en áreas urbanas como la CDMX, están en constante cambio debido al empoderamiento de la mujer y otros factores sociales y económicos (Layder, 2009). Ulloa-Hernández (2017) informa que los contratos sociosexuales en que preferían establecerse los jóvenes eran los de tipo libres y dominantes (independientemente de su sexo); en general las mujeres preferían un compromiso dependiente y los hombres de libertad y dominio; sin embargo, es interesante observar las diferencias de estas preferencias desde el género porque históricamente las mujeres asumían un rol de sumisión, con conceptos de patriarcado y dominación masculina.

Estas nuevas formas de relacionarse en pareja en Latinoamérica y México suelen querer seguir reconociendo algunas tradiciones, pero adaptarse a la modernidad influenciada por la conceptualización del amor en la intimidad, la clase social y el nivel educativo, o sólo a la realidad contextual (DeWall et al., 2011).

En el intento por equilibrar el peso de la tradición y su presencia contrastiva y contradictoria con valoraciones modernas de la sexualidad, según la clase social, el nivel educativo, la dimensión urbano-rural o la identidad de género, se han identificado distintos acuerdos para tener relaciones a distancia (Nuñéz-Noriega y Zazueta-Luzanilla, 2012). Por ejemplo, cada vez es más frecuente y común encontrar parejas que tienen relaciones a distancia. El término "living apart together" (LAT, por sus siglas en inglés) fue creado en Holanda por Michael Berkiel en 1978 (citado en Bernal, 2013); este término significa "vivir juntos, pero separados", es decir, tener una cohabitación intermitente donde los factores que se determinan son normas y pautas en torno a recursos financieros, comunicativos, familiares y sociales, entre otros (Bernal, 2013).

Así, las relaciones de poder entre hombres y mujeres se transforman, según las necesidades por adaptarse al contexto, los cambios sociales y el uso de la tecnología.

Al respecto, las aplicaciones de citas son cada vez un fenómeno más común en el ámbito de las relaciones, ya sea para encontrar una pareja u opciones a una relación actual (Chin, Edelstein y Vernon, 2019). Los millones de usuarios son personas de todo el mundo, lo cual excluye a la ubicación geográfica como limitante para conocer una pareja. En México, encuestas afirman que $42 \%$ de usuarios mexicanos descargaron una aplicación de citas después de terminar una relación (Korkowsky, 2016). Entre las aplicaciones más populares en México se encuentran Tinder, Bumble, Happen y Grindr. La popularidad de éstas ha crecido de manera exponencial en los últimos años, a partir del uso y accesibilidad del smartphone. Sin embargo, algunas aplicaciones ayudan a los usuarios a encontrar pareja u opciones a la misma, mientras que otras ayudan a las parejas ya establecidas que por alguna razón se encuentran a distancia, a permanecer conectadas y comunicadas. Sin duda la tecnología y todos sus derivados son grandes herramientas en la vida de las personas; sin embargo, también incluyen potenciales riesgos. Estudios han demostrado que las aplicaciones, seguidores y likes crean a los usuarios la ilusión de "posibilidades". Personas que se encuentran ya en una relación muestran renuencia a comprometerse más, pues "puede haber alguien mejor; aún hay muchas opciones por explorar". Estas plataformas muestran a los usuarios cuán "accesible" puede ser conocer a alguien más, y todo mediante un "clic". Crean una ilusión de "posibilidades" que impide a las personas el querer comprometerse para seguir estando disponibles (Alvídrez y Rojas-Solís, 2017).

Gracias a Internet, en las dos últimas décadas las redes sociales y las apps para ligar con sus algoritmos que teóricamente incrementan la oferta de potenciales parejas, premian la actividad compulsiva con más visibilidad de los perfiles, y por tanto aumentan la probabilidad de interacción, creando la ilusión de que hay más peces en el mar. Las estadísticas de las aplicaciones de citas así lo prueban: 
el confinamiento incrementó el intercambio de mensajes y la duración de las conversaciones (Vázquez, 2020), se presentó un aumento en las descargas y el número de mensajes en Tinder ha ascendido 30\% (Sierra, 2020).

Estudios recientes sugieren que los comportamientos en y fuera de línea que muestran los jóvenes en las relaciones románticas están estrechamente relacionados. En una investigación con 431 estudiantes universitarios (68.2\% mujeres; de edades de 18 a 26 años, edad promedio 21), que hoy tenían una relación sentimental, se exploró la calidad en línea de sus relaciones románticas y el nivel de satisfacción con dichas relaciones. Los resultados indican que la vida de una pareja también se desarrolla en el espacio de los medios virtuales, y que las dinámicas de relación positivas son más comunes que negativas, y que los hombres jóvenes, más que las mujeres, informaron que coquetean con varias personas mientras están en una relación, destacando el físico como la característica más importante de las personas que conocen en Internet, así como que la influencia en la satisfacción de la relación no es tan importante como otras medidas de la calidad de la relación en línea. Sin embargo, la incursión en el uso de las apps de citas se asocia con la satisfacción en la relación en personas con estilos de apego más inseguros; estos comportamientos reducen la incertidumbre que sienten personas inseguras, lo que a su vez aumenta la satisfacción y seguridad percibida en la relación porque quieren ser el centro de la vida de los demás. En las parejas menos maduras eso indicaría que pueden tener algunos comportamientos en línea que afectan de modo negativo la relación (Sánchez, Muñoz-Fernández y Ortega-Ruiz, 2017).

En esa misma línea, una investigación reciente destacó el perfil y tipo de apego de los usuarios que acuden a las dating apps. Para este estudio se reclutaron 303 adultos estadounidenses que respondieron formularios en línea con reactivos tipo Likert, concluyendo que a partir de dicho perfil y apego se muestran diferentes tipos de interacciones y motivaciones para acudir a estos tipos de aplicaciones. Se analiza cómo el apego y la manera de relacionarse de alguien también se ve reflejada al momento de la interacción digital. Se describen y analizan los perfiles de apego ansioso y evitativo que acuden a esta manera de relacionarse. De acuerdo con los resultados, las personas con apego ansioso son las que más acuden a las aplicaciones de citas. Las personas con este tipo de apego suelen ser muy activas en estas plataformas, y esto se relaciona con el hecho de que se sienten protegidas del rechazo, pues sólo los usuarios que han mostrado interés en ellos les aparecen. Se afirma que la principal motivación para las personas con apego ansioso para utilizar apps de citas es conocer gente en general. A diferencia de las personas con apego evitativo cuya última razón para acceder a estas plataformas era el interés de conocer personas. Las personas con mayor apego evitativo reportaron poca probabilidad de ser usuarios en estas plataformas (Chin et al., 2019).

En otra investigación de corte cualitativo, efectuada en Ciudad de México, donde se pretendió profundizar en la comprensión del uso de las aplicaciones de citas, se trabajó con seis hombres y cinco mujeres, de 30 a 40 años, usuarios de la aplicación de citas Tinder, heterosexuales, que vivían en Ciudad de México, quienes respondieron una entrevista semidirigida. Se exploraron el tipo de dinámicas e interacciones que tienen dichos usuarios en la aplicación, así como sus principales razones y motivaciones para acudir a la misma incluso antes del "ligue" convencional de modo presencial. También se analizó su manera de construir y percibir las relaciones actuales a partir del surgimiento de estas nuevas formas tecnológicas de relación, concluyendo que hay una gran diversidad de motivaciones para acceder a esta aplicación: desde citas y sexo casual hasta búsqueda de relación formal a largo plazo (Korkowsky, 2016).

En el ámbito de las relaciones románticas es evidente la influencia del uso de los dispositivos inteligentes - O smartphones - y las distintas aplicaciones de comunicación. Un estudio del Pew Research Center (citado en Alvídrez y Rojas-Solís, 2017) indica que 27\% de las personas en relación de pareja afirmaron que Internet ha tenido un efecto considerable en su vida de pareja, ya sea de manera positiva o negativa. Pero lo más interesante es que gracias a la popularización de los dispositivos inteligentes (teléfonos celulares o smartphones), al menos 30\% de los encuestados admitió sentir más cercanía o intimidad al intercambiar mensajes de texto con sus respectivas parejas, además de haber solucionado alguna discusión que de otro modo hubiera sido más complicado hacerlo cara a cara. No obstante, otro $33 \%$ de los entrevistados afirmó haberse sentido ignorado por el tiempo que sus parejas le dedican al móvil. En esta investigación el método es una recopilación de diferentes estudios efectuados previamente que permiten delinear y sacar conclusiones de las repercusiones de la tecnología y las aplicaciones de citas en las relaciones románticas actuales. Se concluye que el uso de dispositivos móviles con acceso a Internet o smartphones puede facilitar el inicio de las relaciones románticas por medio de las distintas aplicaciones para microcitas, aunque esto no predice (o garantiza) la permanencia de las mismas. También se observa que las personas tienen diferentes propósi- 
tos relacionales al acceder a las aplicaciones de citas como Tinder; éstas pueden ser desde obtener sexo casual hasta buscar relaciones de largo plazo. Se asocia el intercambio de mensajes de texto en las parejas con el mantenimiento y satisfacción de la relación (Alvídrez y Rojas-Solís, 2017).

\section{Contexto actual: La pandemia por Co-} VID-19

A finales de 2019 comenzó a propagarse el Coronavirus (Covid-19) en la ciudad de Hubei, China, partiendo del virus del síndrome respiratorio agudo severo tipo 2 (SARS-CoV-2) y cuyo tiempo medio de incubación de hasta 14 días, con manifestaciones de tos, fiebre, dificultades para respirar, fatiga, dolor de cabeza y en algunos casos malestares gastrointestinales, mismos que al combinarse con otros padecimientos o factores de riesgo, como diabetes o edad avanzada, pueden llevar hasta a la muerte, con una letalidad de 1 a 3\% (Díaz-Castrillón y Toro-Montoya, 2020). Este virus se expandió a lo largo del mundo llegando a nuestro país a finales de febrero de 2020, Ilevando a la población mexicana a mantenerse en autoconfinamiento desde el 16 de marzo, misma que en la mayoría de los estados se mantiene, exceptuando los comercios esenciales.

Por otro lado, la investigación acerca de las relaciones románticas muestra que los factores de estrés externos, como las dificultades económicas, los trabajos exigentes y los desastres, pueden amenazar la calidad y la estabilidad de las relaciones de pareja (Pietromonaco y Overall, 2021), como es el caso de la actual pandemia por Covid-19. Las reglas de "distanciamiento social" han impuesto límites al sexo por ocio y han creado nuevas oportunidades, como lo muestra una encuesta en línea a 1,559 adultos a quienes se les preguntó respecto al impacto de la pandemia en su vida íntima, y la mitad de la muestra informó una disminución en su vida sexual y la incorporación de nuevas conductas, como el sexting (Lehmiller, Garcia, Gesselman y Mark, 2020). Debido a lo anterior, la vida de las parejas de todo el mundo ha tomado un giro total en cuanto su dinámica, porque ante la necesidad de permanecer en casa las 24 horas del día, han surgido exigencias que requieren de nuevas adaptaciones y desafíos para las mismas, tanto para las que viven juntos, como a las que se han visto forzadas a mantener una relación a distancia debido al autoconfinamiento (Los Ángeles Times, 2020).

Asimismo, este es un evento de vida que sale de la norma y que lleva a toda la población a adquirir nuevas competencias y enfrentar desafíos que no eran necesarios antes, resultando en el surgimiento de conflictos en las parejas al no tener una directriz de cómo actuar ante la nueva situación, misma que además debe equilibrarse con las áreas de funcionamiento (laboral, escolar, emocional, psicológico, social) de los miembros de la pareja, porque éstas también se han visto afectadas y sesgadas (Balzarini et al., 2020).

En una investigación que tuvo como objetivo establecer una relación entre los diferentes estresores generados a raíz del Covid-19 y la calidad y conflictos dentro de la pareja, se aplicó una encuesta vía internet a 3,593 participantes de más de 18 años que se encontraran en una relación romántica, a lo largo de 57 países y en una población mayormente heterosexual ( $77 \%$ mujeres, $23 \%$ hombres). Dicha encuesta medía los reactivos de: aislamiento, finanzas, satisfacción, compromiso, conflicto y sensibilidad de la pareja ante las necesidades del otro; se concluyó que si bien los estresores disminuyen la percepción de satisfacción con la pareja y aumentan el conflicto, la sensibilidad que muestra la pareja parece mitigar el efecto de los estresores, e incluso mejorar la calidad del vínculo (Balzarini et al., 2020).

\section{Posibles efectos en el compromiso}

En el transcurso del tiempo se ha intentado comprender los factores involucrados en el mantenimiento o rompimiento de este vínculo, considerando el nivel de compromiso en la pareja como uno de los principales indicadores de éxito o fracaso, entendiendo éste como el deseo que tiene la pareja por permanecer no sólo con la persona amada, sino también de hacer un esfuerzo considerable en la mejoría y desarrollo de la relación buscando el bienestar conjunto (Nina-Estrella, 2011). Este deseo de permanecer con la persona a través del tiempo se va conformando por las diferentes experiencias, tanto buenas como malas, por las que atraviesan los miembros, mismas que les llevan a cuestionar o reafirmar el deseo y valor que dan a la relación, considerando además los factores intrínsecos y extrínsecos que aporta la pareja (Agnew, Hadden y Tan, 2019).

Para evaluar el efecto en el comportamiento sexual durante la pandemia por Covid-19, Ibarra et al. (2020) efectuaron una encuesta mediante redes sociales a los países de Irán, Italia y España. Este estudio tuvo 279 respuestas, distribuidas entre $58 \%$ de mujeres y $42 \%$ de hombres, midiendo las variables de infidelidad, masturbación, sexting y pornografía. Los resultados indican que, si bien la infidelidad no se vio afectada, la masturbación se incrementó $10 \%$ debido a la necesidad de ais- 
lamiento, así como $16 \%$ en el sexting, entre las parejas que no vivían juntos, además de que sólo $5.5 \%$ de los participantes reportó utilizar apps para citas. El mayor descubrimiento de la presente investigación fue que el consumo de pornografía se incrementó 24\%, (aparentemente después de que la agencia Pornhub liberó su servicio premium mediante la consigna de permanecer en casa durante la pandemia) (Ibarra et al., 2020).

Respecto a lo anterior, es necesario indicar que las dificultades y adversidades vividas dentro de la relación romántica, de no afrontarse de manera adecuada, tienden a disminuir la sensación de satisfacción dentro de la pareja, por lo que, cuando existen opciones externas a la relación tanto de terceras personas como de oportunidades personales en las que no se considera a la pareja y éstas superan el valor subjetivo de la relación, las estadísticas de disolución tienden a aumentar (Torres-González y Ojeda-García, 2009).

Así, el distanciamiento obligado debido a la pandemia por Covid-19 enfrenta a las parejas a diversos estresores: tanto en las que están separadas físicamente, al no tener la gratificación inmediata acostumbrada, como las que viven juntas, pues el convivir las 24 horas con sus áreas de funcionamiento alteradas, genera enojo y frustración, entre otras, mismas que en ocasiones recaen en la pareja.

En la teoría del modelo de inversión propuesto por Rusbult, Martz y Agnew en 1998 se busca establecer las principales variables involucradas en el mantenimiento y duración de las relaciones románticas, encontrando una relación significativa entre los factores de 1) compromiso: el deseo de mantener la relación durante un buen tiempo; 2) satisfacción: la medida en que la relación complace las necesidades de la pareja; 3) inversión: entendido como contribuciones intrínsecas y extrínsecas a la relación, es decir, todo lo que la pareja aporta a la relación como recursos materiales, emocionales y psicológicos, y 4) alternativas: es decir las opciones que tiene la pareja fuera del vínculo romántico para satisfacer sus necesidades. Esta teoría se puso a prueba a partir de la validación de la escala en población mexicana en 2019, concluyendo que es válida y confiable para la población mexicana (Escobar-Mota, Sánchez-Aragón y Lavalle, 2019).

Por lo anterior, los efectos del distanciamiento social debido a la pandemia podrían derivar en un incremento en el uso de la tecnología para fines románticos, con los riesgos que esto implique para el compromiso en la pareja, mostrando un posible aumento en la importancia de las alternativas, por lo que la presente investigación tiene como objetivo explorar si existen diferencias entre el compromiso de pareja antes de la pandemia y en la actualidad, y si estas diferencias están relacionadas con el uso de redes sociales por jóvenes universitarios en el contexto de la pandemia por Covid-19. La presente investigación en relaciones interpersonales contribuye al cuerpo de análisis en el contexto de una emergencia sanitaria mundial planteando nuevos cuestionamientos acerca del compromiso, el distanciamiento físico y el acercamiento digital, y su función en la formación de relaciones sólidas que contribuyan a una vida larga, saludable y satisfactoria.

\section{MÉTodo}

\section{Participantes}

Para la presente investigación se trabajó con una muestra no probabilística, por cuota, de 391 participantes voluntarios y anónimos de nacionalidad mexicana, con un promedio de edad de 23 años en un rango entre los 18 y 48 años. Dicha muestra estaba conformada por $56 \%$ de mujeres y $44 \%$ de hombres, de los cuales $82 \%$ tenían un nivel de licenciatura y $18 \%$ de posgrado, y más de la mitad (56\%) de la muestra fueron estudiantes de la Universidad Anáhuac y con dos años y tres meses de duración en la relación en promedio donde sólo 16\% vivía con su pareja.

El tamaño de la muestra fue adecuado considerando el error máximo admisible y el nivel de confianza asociado a dicha estimación, dada la heterogeneidad de la población y los procedimientos de análisis a utilizar (Cohen, 1992). Todos los participantes debían estar en una relación de pareja con al menos un año de duración y proporcionar un consentimiento informado para proteger los derechos y garantías de los mismos.

\section{Materiales}

1. Cuestionario con preguntas que exploran variables sociodemográficas (serie de preguntas relevantes a los objetivos del estudio).

2. Cuestionario con preguntas que exploran comportamientos en línea y fuera de línea con intenciones romántico sexuales/románticas con el apoyo de las tecnologías de la información (serie de preguntas relevantes a los objetivos del estudio, creadas a partir de investigaciones previas en comportamientos en línea, sin necesidad de formar un constructo independiente).

3. Escala del modelo de inversión (Escobar-Mota et al., 2019) compuesta por cuatro factores: 1) compromiso: deseo de mantener la relación de pareja durante largo tiempo, así como enfocarse en dicha meta, pues ello contribuye al sentimiento de unión 
y apego con el otro; 2) satisfacción: la medida en que la relación de pareja complace las necesidades de intimidad, compañerismo y hace feliz a la persona; 3) alternativas: percepción que tiene la persona de las opciones posibles de personas que pudieran satisfacer las necesidades de intimidad y compañerismo de no contar con su pareja, y 4) inversión: aspectos que han sido contribuciones personales a la relación, como amigos, deudas, tiempo y bienes materiales, que se verían amenazados si el vínculo terminara. Los cuatro factores en conjunto explican $62.79 \%$ de la varianza con un Alpha de Chronbach de 0.73 , confirmando que la escala es confiable y previamente validada en población mexicana. Los mexicanos identifican con claridad la diferencia entre la satisfacción que proporciona una relación de pareja, las inversiones a la misma y las alternativas a ésta, y así se manifiesta el compromiso a mantenerse dentro de ella y, por ende, representa una herramienta útil en la medición de uno de los constructos de mayor relevancia en el entendimiento de las relaciones de pareja: el compromiso.

\section{Procedimiento}

Las preguntas se adaptaron a un cuestionario digital por medio de la plataforma Google Forms. Se contactó a los participantes vía redes sociales y se solicitó respondieran de manera anónima. El cuestionario estuvo disponible durante tres meses. Con el apoyo de la plataforma SPSS V.19, se probaron los criterios de normalidad y homogeneidad, se confirmaron los criterios y características de inclusión y una prueba t de Student para muestras independientes considerando el tiempo anterior a la pandemia y la actualidad; por último, se hizo un análisis de correlación de Pearson producto momento para determinar la asociación de las variables.

\section{Mediciones}

Las variables que se consideraron para el estudio fueron el compromiso, satisfacción, alternativas e inversión antes de la pandemia y en la actualidad, así como el uso de redes sociales; esto se hizo por medio de la aplicación de un cuestionario de varibles sociodemográficas, un cuestionario de comportamiento en línea y la escala del modelo de inversión (Escobar-Mota et al., 2019)

\section{Análisis estadísticos}

Se hizo un análisis de frecuencias para descartar errores, comprobar criterios de inclusión y conocer las características descriptivas de los participantes, así como una comparación entre índices de niveles de respuesta en la escala Likert determinada de 1-5, para describir el patrón general de respuesta antes y durante la pandemia. Luego se plateó un diseño no experimental, comparativo, con un enfoque temporal retrospectivo (Ato, López y Benavente, 2013) por medio de una prueba $T$ para muestras relacionadas; como complemento se hizo un análisis de correlaciones bivariadas entre todas las variables para conocer la fuerza de asociación entre las mismas.

\section{Resultados}

Se comparó el índice de participantes que respondieron en los niveles 4 y 5 de la escala Likert para los reactivos relacionados con el compromiso de pareja (alternativas, compromiso, satisfacción e inversión) antes de la pandemia y en la actualidad, obteniendo que antes de la pandemia $81.07 \%$ de los encuestados se enfocaban en un futuro a largo plazo en su relación, mientras que en la actualidad sólo $65 \%$, que antes $83.9 \%$ se sentía muy unida y apegada a su pareja y hoy esta cambió a $60.1 \%$, mientras que la satisfacción en la relación cambió de $86.7 \%$ a $59.9 \%$, así como la percepción de ser felices en la relación que correspondía a $91 \%$ de la muestra y ahora corresponde a $68.6 \%$. También se observó que antes de la pandemia $82.8 \%$ reportó que su relación satisfacía sus necesidades de intimidad, compañerismo, etcétera, en tanto que ahora este índice cambió a 54.3\%. En cuanto a la inversión, $65.5 \%$ de las personas encuestadas expresó haber puesto mucho en la relación, lo cual se perdería si la relación terminara, mientras que ahora este es de $61.6 \%$, además de que antes sólo $23.1 \%$ consideraba tener excelentes opciones adicionales a su relación, aumentando a $37.9 \%$, donde $75.4 \%$ consideraba que sus necesidades de intimidad y compañerismo no podrían ser satisfechas fácilmente por otra persona, disminuyendo a $55.3 \%$. Se mostró también que antes de la pandemia sólo 7.7\% de los participantes no se sentirían demasiado afectados si la relación con su pareja terminara, aumentando a 16\%, y que antes sólo 5.6\% estaba de acuerdo ante la idea de tener una nueva pareja en el siguiente año, mientras que en la actualidad esta cifra aumentó a 22.6\%.

Referente al uso de redes sociales, la mayoría de los participantes (83.39\%) expresaron estar en desacuerdo con que a partir de la pandemia han utilizado más aplicaciones sociales, en contraste con $21.2 \%$, quienes expresaron estar de acuerdo con que a partir de la pandemia conocieron a más personas en línea, y sólo $12.3 \%$ ha coqueteado con más personas en las redes sociales. Se observa también que sólo $10 \%$ de los participantes mostró acuerdo con que a partir de la pandemia se han involucrado emocional o afectivamente con alguien ajeno a la relación, mientras 
que $16.1 \%$ reportó haberse involucrado sexualmente con alguien más, y 5.4\% haber iniciado una relación romántica con alguien que no fuera su pareja.

Al comparar las medias de las variables de compromiso, antes de la pandemia y en la actualidad, se encontraron diferencias estadísticamente significativas en tres de ellas (satisfacción, compromiso y alternativas), mientras que uso de redes sociales y lo invertido en la relación se mantiene sin cambios (tabla 1 ).

Tabla 1.

Diferencias en alternativas, compromiso, satisfacción e inversiones

\begin{tabular}{|c|c|c|c|c|c|}
\hline \multirow{2}{*}{ Medida } & \multicolumn{2}{|c|}{$\begin{array}{l}\text { ANTES DE LA } \\
\text { PANDEMIA }\end{array}$} & \multicolumn{2}{|c|}{ ACtUALMENTE } & \multirow{2}{*}{$t$} \\
\hline & $\mathbf{M}$ & DE & M & DE & \\
\hline Alternativas & 1.97 & 0.898 & 2.42 & 1.18 & $-7.3^{* *}$ \\
\hline Compromiso & 4.36 & 0.759 & 3.79 & 1.19 & $10.5^{* *}$ \\
\hline Satisfacción & 4.13 & 0.774 & 3.48 & 1.33 & $10.8^{* *}$ \\
\hline Inversión & 3.60 & 0.970 & 3.61 & 1.02 & -0.227 \\
\hline${ }^{* *} p<0.01$. & & & & & \\
\hline
\end{tabular}

Se muestra una diferencia a favor de las alternativas actualmente, es decir, que hay mayor percepción de alternativas atractivas a la pareja en la actualidad, una me- nor percepción de compromiso y una menor percepción de satisfacción con la pareja, que antes de la pandemia. Respecto a la variable de inversión se puede concluir que no hay una diferencia significativa entre el antes y el después, es decir, que no parece haber cambiado la percepción de lo que se ha invertido en la relación.

Asimismo, por medio de una correlación de Pearson exploramos la relación y significancia de las variables (tabla 2).

Se encontró una correlación positiva entre las variables de redes sociales y alternativas antes (0.16), y aún mayor en la percepción de alternativas actual (0.43), y esta es inversamente proporcional a las variables de compromiso, satisfacción e inversión actual, es decir, que al utilizar redes sociales las alternativas aumentan y disminuye la relación con compromiso, la satisfacción y la inversión en la relación. Por tanto, se puede concluir que ante el aumento del uso de redes sociales y la percepción de alternativas disminuye el nivel de compromiso, mientras que con el aumento de satisfacción e inversión también lo hace el compromiso. En la variable de inversión antes de la pandemia, puede verse una correlación negativa con alternativas antes y positiva con compromiso antes y satisfacción antes; esto es, que a menor inversión antes de la pandemia, no había tanta percepción de alternativas, mientras que había mayor compromiso y satisfacción.

\section{Tabla 2.}

Intercorrelaciones, medias y desviaciones estándar para las variables de la escala del modelo de inversión (antes de la pandemia y actualmente) y para la variable de redes sociales.

\begin{tabular}{|c|c|c|c|c|c|c|c|c|c|c|c|}
\hline SUbEscalas & 1 & 2 & 3 & 4 & 5 & 6 & 7 & 8 & 9 & $M$ & DE \\
\hline $\begin{array}{l}\text { Redes } \\
\text { sociales }\end{array}$ & - & & & & & & & & & 1.5 & 1.0 \\
\hline $\begin{array}{l}\text { Alternativas } \\
\text { antes }\end{array}$ & $0.164^{* *}$ & - & & & & & & & & 1.9 & 0.8 \\
\hline $\begin{array}{l}\text { Alternativas } \\
\text { ahora }\end{array}$ & $0.434^{* *}$ & $0.350^{* *}$ & - & & & & & & & 2.4 & 1.1 \\
\hline $\begin{array}{l}\text { Compromiso } \\
\text { antes }\end{array}$ & $-0.279^{* *}$ & $-0.318^{* *}$ & $-0.323^{* *}$ & - & & & & & & 4,3 & 0.7 \\
\hline $\begin{array}{l}\text { Compromiso } \\
\text { ahora }\end{array}$ & $-0.521^{* *}$ & $-0.144^{* *}$ & $-0.681^{* *}$ & $0.465^{* *}$ & - & & & & & 3.7 & 1.1 \\
\hline $\begin{array}{l}\text { Satisfacción } \\
\text { antes }\end{array}$ & $-0.302^{* *}$ & $-0.140^{* *}$ & $-0.283^{* *}$ & $0.683^{* *}$ & $0.445^{* *}$ & - & & & & 4.1 & 0.7 \\
\hline $\begin{array}{l}\text { Satisfacción } \\
\text { ahora }\end{array}$ & $-0.466^{* *}$ & -0.024 & $-0.643^{* *}$ & $0.374^{* *}$ & $0.900^{* *}$ & $0.464^{* *}$ & - & & & 3.4 & 1.3 \\
\hline $\begin{array}{l}\text { Inversión } \\
\text { antes }\end{array}$ & 0.001 & $-0.248^{* *}$ & -0.031 & $0.383^{* *}$ & 0.049 & $0.253^{* *}$ & 0.024 & - & & 3.6 & 0.9 \\
\hline $\begin{array}{l}\text { Inversión } \\
\text { ahora }\end{array}$ & -0.235 & $-0.278^{* *}$ & $-0.266^{* *}$ & $0.222^{* *}$ & $0.398^{* *}$ & $0.181 * *$ & $0.345^{* *}$ & $0.563^{* *}$ & - & 3.6 & 1.0 \\
\hline
\end{tabular}




\section{Discusión}

Los resultados de la presente investigación permiten conocer más acerca del papel del distanciamiento físico involuntario (debido a la pandemia por Covid-19) y el uso de redes sociales en el compromiso de las parejas, de modo que este estudio permitió evaluar los cambios que se dieron en el compromiso de las parejas antes del distanciamiento y durante éste.

Las limitaciones del estudio podrían ser que los participantes requerían del uso de su memoria para recordar cuál era su percepción del compromiso porque no se aplicaron los instrumentos con anterioridad, si no que en el mismo cuestionario debían responder cómo se sentían antes de la pandemia por Covid-19 y cómo se sentían durante la misma.

El compromiso y la satisfacción en la pareja disminuye antes y después del distanciamiento debido a la pandemia. Derivado de lo anterior, la percepción de opciones atractivas a la pareja actual aumenta, y esto se relaciona con el uso de tecnologías de la información.

Al respecto, Alvídrez y Rojas-Solís (2017) explicaron que las aplicaciones, seguidores y "likes" le dan a los usuarios una ilusión de posibilidades. Frente esto las personas son menos propensas a comprometerse porque mantienen la posibilidad de "encontrar a alguien mejor", ya que con estas aplicaciones el usuario tiene a un clic la oportunidad de conocer a alguien más. A su vez, Agnew et al. (2019) mencionan que con el paso del tiempo hay una menor percepción de compromiso que antes, debido que el deseo de permanecer con la pareja y las situaciones que se les puedan presentar, tanto buenas como malas, llevan a las personas a cuestionarse la intención de continuar con la relación.

Respecto al papel de la pandemia, Torres-González y Ojeda-García (2009) explican que si una pareja se enfrenta a dificultades y adversidades cuando no se sobreIlevan de manera adecuada, la sensación de satisfacción dentro de la pareja disminuye, y cuando hay opciones externas a la relación y éstas superan el valor subjetivo de la misma, se incrementa el índice de la disolución de parejas. En esta línea, el estudio de Sánchez et al. (2017) confirma que los hombres jóvenes, más que las mujeres, continúan coqueteando con otras personas en internet mientras están en una relación amorosa, y esto puede tener un efecto en la satisfacción de la relación que se está manteniendo y en el compromiso de la misma.

Por otro lado, el uso de redes sociales está relacionado con la percepción de las alternativas porque estas aplicaciones relacionadas con citas cada vez son más usadas, debido a que facilitan encontrar pareja u opciones a una relación actual (Chin et al., 2019). Incluso proporcionan la idea de excluir la ubicación geográfica como limitante para que las personas conozcan una pareja. Según Korkowsky (2016), en México los usuarios de estas aplicaciones recurren a ellas después de la ruptura amorosa.

En cuanto a las inversiones que son entendidas como contribuciones intrínsecas y extrínsecas a la relación, se observa que los resultados no fluctuaron entre el antes y después de la pandemia. Esto pudiera deberse a que la edad promedio de la muestra es de 23 años, con un promedio de dos años de duración en la relación, donde sólo $16 \%$ vivía con su pareja, por lo que es posible inferir que la mayoría de estas parejas tenía, antes de la pandemia, cierta inversión en su relación, pero no tan determinante como hijos o una hipoteca, y al Ilegar la pandemia, dado que no eran relaciones muy largas o con planes a corto plazo de formación de familias, por ejemplo, la pandemia hizo que todos los planes y metas al futuro se pausaran, pero no disminuyera, pues todos esperaban en un futuro próximo volver a la normalidad.

\section{Conclusiones}

Los hallazgos de este estudio corroboran trabajos anteriores que demuestran el poderoso papel que desempeña la desconexión social, física y psicológica, en combinación con el uso de las tecnologías de la comunicación (redes sociales) y su impacto en el compromiso de las relaciones de pareja en el contexto de la modernidad, entendida como cambios en el rol de las mujeres y hombres, y una economía frágil y cambiante. Se invita a la reflexión de resignificar la libertad como aquella en que el contexto, los medios y los involucrados compartan una responsabilidad asociada a su libre albedrío y autonomía, así como a condiciones de equidad y reciprocidad para negociar decisiones y objetivos tendientes a procurar un empoderamiento mutuo (Ulloa-Hernández, 2017), porque en todo caso, como indica Esteinou (2008), es tarea de todos entender que la modernidad tiene muchos rostros y que los procesos "tradicionales" y "modernos" confluyen de manera compleja ante nuevas formas de organización social, pero que también confluyen de modo complejo en la configuración de las subjetividades y de la vida íntima de hombres y mujeres.

Se sugieren análisis adicionales para contrastar entre parejas con distintos acuerdos de exclusividad y/o en participantes con características demográficas más apegadas a la población en general. 


\section{Referencias}

Anderson, D. R., Sweeney, D. J., \& Williams, T. A. (1999). Estadística para administración y economía. México: CENGAGE Learning.

Baum, S., \& Steele, P. (2017). Who Goes to Graduate School and Who Succeeds? (Vol. null).

Blalock, H. M. (1978). Estadística social. México: Fondo e Cultura Económica.

Carter, J., Brown, M., \& Simpson, K. (2017). From the Classroom to the workplace: how social science students are doing data analysis for real. Statistics Education Research Journal, 16(1), 80-101.

Casas-Rosal, J. C., Caridad y Ocerín, J. M., Núñez-Tabales, J. M., \& León-Mantero, C. (2019). Teaching statistics through the Real Estate Data Analyzer software. Teaching Statistics, 41 (2), 58-64. doi: $10.1111 /$ test. 12183

Cobb, G. W., \& Moore, D. S. (1997). Mathematics, Statistics, and Teaching. The American Mathematical Monthly, 104 (9), 801-823. doi: $10.2307 / 2975286$

Cvetkovic-Vega, A., Inga-Berrospi, F., \& Mestas, C. A. (2017). Organizaciones científicas estudiantiles como semilleros de líderes y gestores de la investigación científica en el Perú: SOCIMEP. Acta Médica Peruana, 34(1), 70-71.

Daniel, W. W. (1977). Bioestadística : Base para el análisis de las ciencias de la salud México: LIMUSA-Noriega.

Eudave Muñoz, D., Carvajal Ciprés, M., \& Páez, D. A. (2018). Importancia y utilidad de la estadística en profesiones del campo de las ciencias sociales. RECIE. Revista Electrónica Científica de Investigación Educativa, 4(1), 283-293.

GAISE-College-Report. (2016). Guidelines for Assessment and Instruction in Statistics Education College Report 2016. http://www.amstat.org/education/gaise.

Glass, G. V., \& Stanley, J. C. (1974). Métodos estadísticos aplicados a las ciencias sociales: Prentice Hall Hispanoamericana S. A.

Gómez-Restrepo, C. (2017). Semilleros de investigación: impulso a la innovación, el aprendizaje y la docencia. Universitas Médica, 58(1).

Guerra-Molina, R. (2017). ¿ Formación para la investigación o investigación formativa?. La investigación y la formación como pilar común de desarrollo. Revista Boletín Redipe, 6(1), 84-89.

Guerrero-Useda, M. E. (2007). Formación de habilidades para la investigación desde el pregrado. Acta colombiana de psicología, 10(2), 190-192.

Hernández-Sampieri, R., Fernández-Collado, C., \& BaptistaLucio, M. d. P. (2014). Metodología de la investigación. México: McGraw Hill.

Immekus, J. C. (2019). Flipping Statistics Courses in Graduate Education: Integration of Cognitive Psychology and Technology. Journal of Statistics Education, 27(2), 79-89. doi: $10.1080 / 10691898.2019 .1629852$

Jones, R. C. (2019). Statistical investigation measuring intelligence and creativity. Teaching Statistics, 41(1), 3640. doi: 10.1111/test.12169

Kerlinger, F. (1975). Investigación del comportamiento. Técnica y metodo. México: NO SE.

Lord, F. M. (1953). On the Statistical Treatment of Football
Numbers. American Psychologist, 8(12), 750-751. doi: 10.1037/h0063675

Loy, A., Kuiper, S., \& Chihara, L. (2019). Supporting Data Science in the Statistics Curriculum. Journal of Statistics Education, 27(1), 2-11. doi: 10.1080/10691898.2018.1564638

MacGillivray, H. (2019). Statistics and data science are NOT branches of mathematics-or of any other discipline. Teaching Statistics, 41(2), 41-41. doi: 10.1111/test.12197

Manzano-Patiño, A., \& Zamora-Muñoz, S. (2009). Sistema de ecuaciones estructurales: una herramienta de investigación. México: Centro Nacional de Evaluación para la Educación Superior, A. C. .

McKensey\&Company. (2011). Big data: The next frontier for innovation, competition, and productivity. https:// bigdatawg.nist.gov/pdf/MGl big_data_full_report.pdf

Montoya, J., \& Peláez, L. E. (2013). Investigación formativa e investigación en sentido estricto: una reflexión para diferenciar su aplicación en instituciones de educación superior. Entre Ciencia e ingeniería, 13, 20-25.

Nerlove, M., Grether, D. M., \& Carvalho, J. L. (1988). Análisisa de series temporales económicas. México: Fondo de Cultura Económica.

Numa-Sanjuan, N., \& Márquez-Delgado, R. A. (2019). Los semilleros como espacios de investigación para el investigador novel. Propósitos y Representaciones, 7, 230239. doi: 10.20511/pyr2019.v7n1.289

Pardinas, F. (1969). Metodología y técnicas de la investigación en ciencias sociales. México: Siglo XXI.

Payne, G., Williams, M., \& Chamberlain, S. (2004). Methodological pluralism in British sociology. Sociology, 38(1), 153-163.

Rodríguez-Morales, A., Milanés-Gómez, R., \& Avila-Portuondo, A. M. (2016). La profesionalización una fuente para semilleros de investigación. Revista Conrado, 12(55), 83-89.

Rojek, C., \& Turner, B. (2000). Decorative Sociology: Towards a Critique of the Cultural Turn. The Sociological Review, 48(4), 629-648. doi: 10.1111/1467-954x.00236

Roy, S. (2019). Inferential statistics made fun: stories that boxes of '100 paper clips' can tell. Teaching Statistics, 41(1), 25-29. doi: $10.1111 /$ test. 12168

Saavedra-Cantor, C. J., Antolínez-Figueroa, C., Puerto-Guerrero, A. H., Muñoz-Sánchez, A. I., \& Rubiano-Mesa, Y. L. (2015). Semilleros de investigación: desarrollos y desafíos para la formación en pregrado. Educación y educadores, 18(3), 391-407.

Silva-Rodriguez, A. (1992). Metodos cuantitativos en psicología. Un enfoque metodológico. México: Trillas.

Songsore, E., \& White, B. J. (2018). Students' perceptions of the future relevance of statistics afer completing an online introductory statistics course. Statistics Education Research Journal, 17(2), 120-140.

Spiegel, M. R. (1970). Estadística. México: MacGraw-Hill.

Spiegel, M. R. (1977). Probabilidad y estadística. México: MacGraw-Hill.

Strayer, J. F., Gerstenschlager, N. E., Green, L. B., McCormick, N., MaDaniel, S., \& Rowell, G. H. (2019). Toward a full(er) implement of active learning. Statistics Education Research Journal, 18(1), 63-82.

Tovar-Torres, C. (2018). Investigación formativa. Perspectivas(11). Villalba-Cuéllar, J. C., \& González-Serrano, A. (2017). La importancia de los semilleros de investigación. Revista 
Prolegómeno, 20(39), 9-10.

Wesbster, A. L. (2000). Estadística aplicada a los negocios y a la economía. México: MacGraw-Hill.

Williams, M., Payne, G., Hodgkinson, L., \& Poade, D. (2008). Does British Sociology Count? Sociology Students' Attitudes toward Quantitative Methods. SAGE, 42(5), 1003-1021.

Zapata-Cardona, L., \& González-Gómez, D. (2017). Imágenes de los profesores sobre la estadística y su enseñanza. Educación matemática, 29, 61-90.

Zapata-Peña, J., Ortiz-Puerto, A. C., Bautista-Rincón, R., DíazGonzález, J., Tinoco, L. A., \& Ayala-Cortés, C. (2014). Una experiencia sobre la conformación de semilleros de investigación en la formación inicial de profesores. Revista EDUCYT, 9, 1-9. 


\section{Dimensión Cuantitativa}

\section{Perfil de Evaluación entre pares}
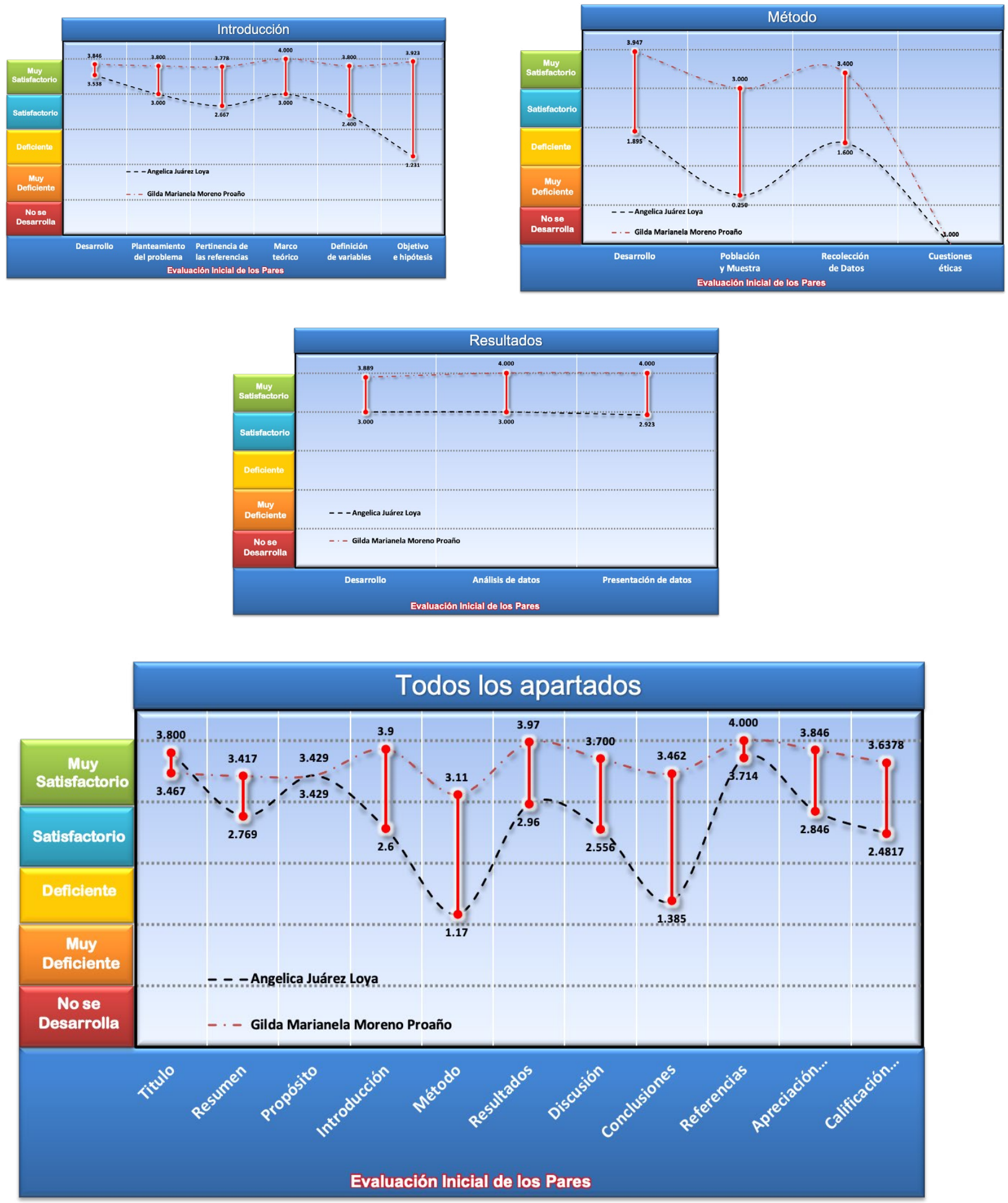


\section{Índice de Concordancia}

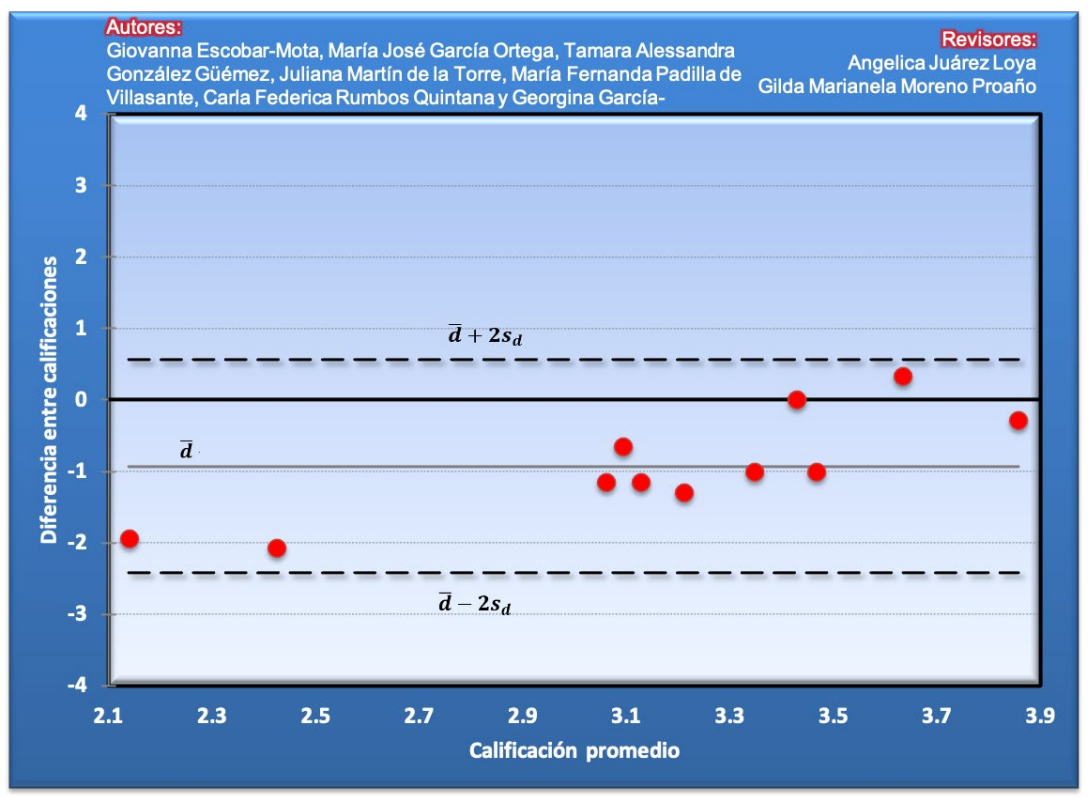

\section{Índice de Acuerdo}

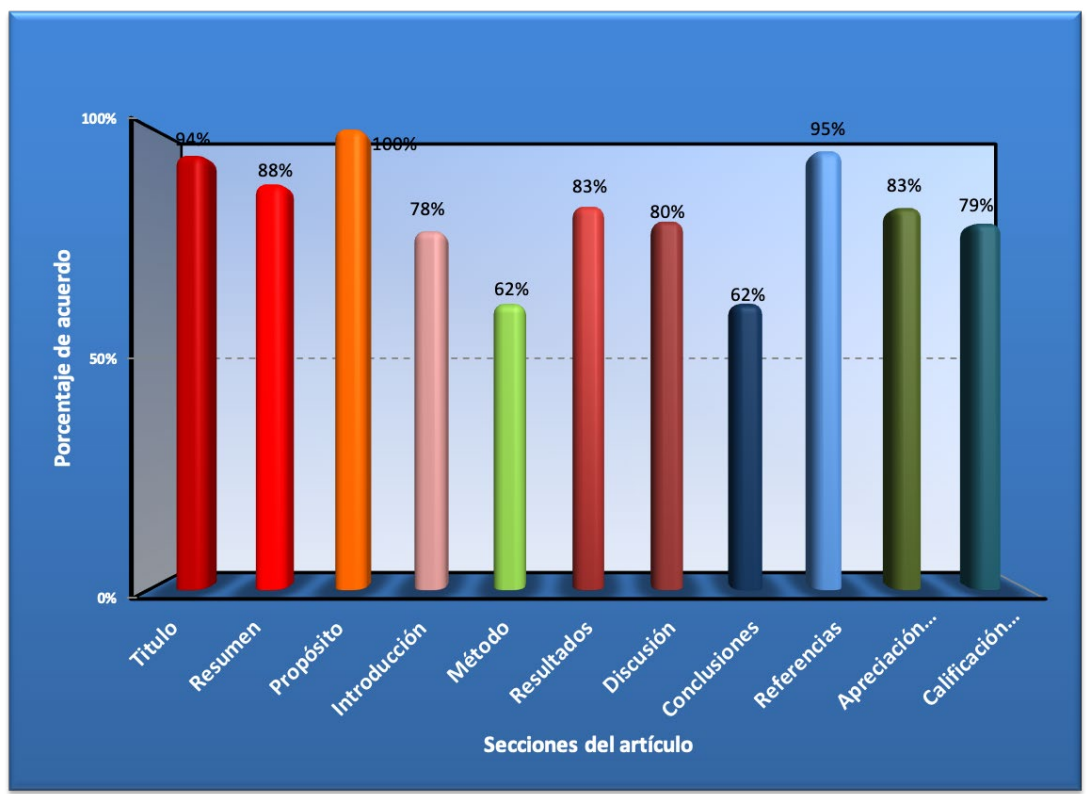




\begin{tabular}{|c|c|}
\hline Revisor 1 & Revisor 2 \\
\hline Angélica Juárez Loya & Gilda Marianela Proaño \\
\hline \multicolumn{2}{|c|}{ Título/Autoría } \\
\hline $\begin{array}{l}\text { Este título puede prescindir de los artículos para evitar su- } \\
\text { perar las } 15 \text { palabras máximas. }\end{array}$ & $\begin{array}{l}\text { No se comprende la descripción "compromiso en la pare- } \\
\text { ja" Falta explicar durante cuál pandemia. }\end{array}$ \\
\hline \multicolumn{2}{|c|}{ Resumen } \\
\hline $\begin{array}{l}\text { - El resumen debe tener como máximo } 150 \text { palabras y } \\
\text { estos resúmenes superan las } 200 \text { palabras (en español y } \\
\text { en inglés). - No se incluyen al menos } 5 \text { palabras clave y } \\
\text { en la última palabra clave ponen la palabra y antes de } \\
\text { enunciar "apps de citas", lo cual es incorrecto, cuidar } \\
\text { este detalle, no se ponen y o and en las palabras clave o } \\
\text { keywords. }\end{array}$ & $\begin{array}{l}\text { El resumen se compone de más de } 150 \text { palabras. No se } \\
\text { explica con claridad la intención del estudio. No se com- } \\
\text { prende si una de las variables de estudio son las TIC o las } \\
\text { redes sociales. El término "inversión", no explica a qué } \\
\text { tipo de inversión se refiere, si financiera, de tiempo, etc. } \\
\text { En las palabras clave aparece apps de citas; sin embargo, } \\
\text { sería mejor escribir redes sociales. }\end{array}$ \\
\hline \multicolumn{2}{|c|}{ Próposito del Estudio } \\
\hline $\begin{array}{l}\text { Aunque se enuncia el propósito de forma clara y es } \\
\text { congruente con el diseño y análisis de resultados, no } \\
\text { se incluye la justificación del estudio o lo relevante que } \\
\text { será para la comunidad científica. }\end{array}$ & $\begin{array}{l}\text { Falta describir el propósito del estudio y que se conecte } \\
\text { con los resultados obtenidos. }\end{array}$ \\
\hline \multicolumn{2}{|c|}{ Introducción } \\
\hline $\begin{array}{l}\text { - Será factible que hagan una descripción pormenoriza- } \\
\text { da de las variables que estudiaron, no es suficiente con lo } \\
\text { que describieron en la sección de materiales o mediciones } \\
\text { puesto que no se definen dichos constructos. - No descri- } \\
\text { ben sus hipótesis de forma clara, la revista solicita que se } \\
\text { realice esta actividad. }\end{array}$ & $\begin{array}{l}\text { Actualizar bibliografía desde la aparición de la pandemia } \\
\text { del Covid-19. }\end{array}$ \\
\hline
\end{tabular}




\begin{tabular}{|c|c|}
\hline Revisor 1 & Revisor 2 \\
\hline \multicolumn{2}{|c|}{ Método } \\
\hline $\begin{array}{l}\text { - Los autores no desarrollan las condiciones éticas y de } \\
\text { cuidado de los participantes que serían necesarias a la } \\
\text { hora de recopilar datos personales e información de los } \\
\text { paticipantes. - En la descripción de materiales, es insufi- } \\
\text { ciente la explicación que realizan, principalmente de los } \\
\text { dos primeros cuestionarios, en los cuales no mencionan } \\
\text { si tienen propiedades psicométricas o cómo fueron dise- } \\
\text { ñados y en el caso de la Escala del Modelo de Inversión, } \\
\text { no explican el objetivo de medición de dicho instrumen- } \\
\text { to. - No realizan una descripción del diseño del estudio y } \\
\text { tampoco del muestreo. - El procedimiento no deja ver de } \\
\text { qué manera se contactó a los participantes y no deja ver si } \\
\text { tuvieron los cuidados éticos para el tratamiento de datos } \\
\text { personales o los posibles riesgos que se asumen en una } \\
\text { investigación como esta. En dicha sección confunden la } \\
\text { descripción de análisis estadísticos con el proceso para } \\
\text { llevar a cabo la investigación. - No definen conceptual- } \\
\text { mente las variables incluidas en el estudio. - Enuncian } \\
\text { el procedimiento de análisis, pero no dicen claramente } \\
\text { todas las pruebas estadísticas utilizadas (p. ej. es me- } \\
\text { jor enunciar completamente "prueba t de Student para } \\
\text { muestras independientes"), no mencionan si probaron } \\
\text { los supuestos estadísticos necesarios para la elección de } \\
\text { sus pruebas (normalidad, homogeneidad de varianzas, } \\
\text { etc.) y tampoco mencionan con qué programa estadísti- } \\
\text { co y versión del programa realizaron sus análisis. }\end{array}$ & $\begin{array}{l}\text { Falta explicar el tipo de muestra escogido. No se mencio- } \\
\text { nan los aspectos éticos. }\end{array}$ \\
\hline
\end{tabular}

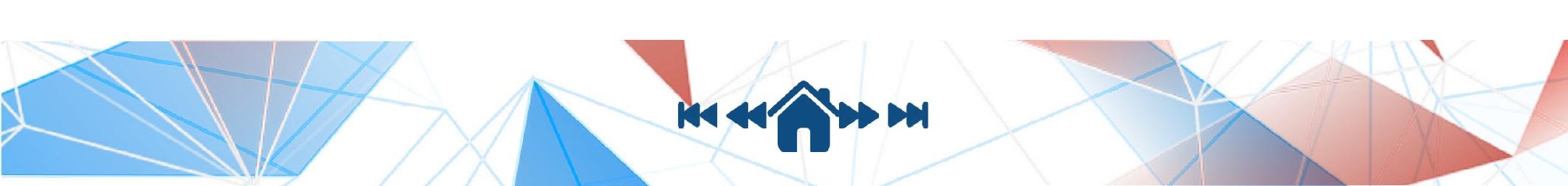




\begin{tabular}{|c|c|}
\hline Revisor 1 & Revisor 2 \\
\hline \multicolumn{2}{|c|}{ Resultados } \\
\hline $\begin{array}{l}\text { - En la descripción de resultados, pudieron elaborar una } \\
\text { tabla de estadísticos descriptivos en lugar de solo enun- } \\
\text { ciarlos en sus resultados. Los datos descriptivos descritos, } \\
\text { no indican por qué elegir solo la comparación de los pun- } \\
\text { tajes } 4 \text { y } 5 \text { de las escalas Likert de sus variables. Se ten- } \\
\text { dría que justificar por qué hicieron esto. - No indican si } \\
\text { probaron los supuestos para utilizar estadística paramé- } \\
\text { trica. - En su tabla 1, no siguen la secuencia de asteriscos } \\
\text { en la nota, se puede utilizar solo un asterisco para indicar } \\
\text { a qué nivel estuvo definido el criterio de p, puesto que no } \\
\text { tienen numerales que utilicen la primera indicación (un } \\
\text { solo asterisco). - Es hasta esta sección que se indica que } \\
\text { se utilizó correlación de Pearson, pero no se había dicho } \\
\text { en la sección de análisis estadísticos. - En el cuerpo del } \\
\text { texto, cuando se indica un hallazgo, correlación con sig- } \\
\text { nificancia, etc. se debe poner entre paréntesis los símbolos } \\
\text { y valores de cada variable (p. ej. si se encontró correlación } \\
\text { entre variables, se tendría que escribir: } r=. x x x ; p=x x x ; \\
\text { otro ejemplo es con la prueba t, se tendría que poner: } t=x \text {. } \\
x x ; \text { gl=x; } p=. x x x \text { ) }\end{array}$ & Los resultados son claros \\
\hline \multicolumn{2}{|c|}{ Discusión } \\
\hline $\begin{array}{l}\text { - Aunque pusieron énfasis desde el título en la pobla- } \\
\text { ción universitaria, no se argumenta lo suficiente en los } \\
\text { hallazgos de esta población en específico, las investiga- } \\
\text { ciones que presentan están más centradas en población } \\
\text { general. - No dejan ver la utilidad de sus hallazgos para } \\
\text { la comunidad científica, algo que está limitado desde su } \\
\text { planteamiento. - No mencionan cuáles pueden ser las } \\
\text { limitaciones de la investigación. }\end{array}$ & La discusión al inicio de los resultados propios es escasa. \\
\hline
\end{tabular}




\section{Revisor 1}

Revisor 2

\section{Conclusiones}

- Me parece importante que sus conclusiones coincidan con los hallazgos de su investigación, parecen no estar basadas en los datos que encontraron, por ejemplo en la siguiente frase: "se invita a la reflexión de resignificar la libertad, como aquella en que el contexto, los medios y los involucrados, compartan una responsabilidad asociada a su libre albedrío y autonomía, así como a condiciones de equidad y reciprocidad para negociar decisiones y metas, tendientes a procurar un empoderamiento mutuo (Ulloa Hernández, 2017) dado que en todo caso, como señala, Esteinou, (2008) es tarea de todos, entender que la moderNo se señalan las deficiencias o dificultades del estudio; por ejemplo, el considerar el contexto de la pandemia del Covid-19. nidad tiene muchos rostros y que los procesos «tradicionales» y «modernos» confluyen de manera compleja ante nuevas formas de organización social, pero que también confluyen de manera compleja en la configuración de las subjetividades y de la vida íntima de hombres y mujeres. “ - Vuelven a omitir a la población universitaria como foco primordial de su investigación.

\section{Referencias}

- Su lista de referencias y citación debe coincidir con un solo formato APA, tienen una combinación de APA 6ta ed. y APA 7a ed. Según revisé en los criterios de la revista aún solicitan APA 6ta ed., revisar puesto que no todo coincide.

no hay sugerencias de cambio 


\section{EDITORIAL}

